\title{
REAL-TIME CONTOUR HOLOGRAPHY USING BSO CRYSTALS
}

\author{
F.M. KÜCHEL and H.J. TIZIANI \\ Institut für Technische Optik der Universität Stuttgart, \\ 7000 Stuttgart 80, Fed. Rep. Germany
}

Received 17 March 1981

\begin{abstract}
Contour holography can be a powerful tool for contactless measurements. Its application in real time is even more useful for the engineer. The BSO crystal was found to be appropriate for real-time contour holography using two wavelengths.
\end{abstract}

\section{Introduction}

Contour-line holography as described by Hildebrand, Varner and others [1-4] is a useful tool for contactless measurements of contours of objects with optically rough surfaces. Applying the technique in real time is even more attractive and leads to an easier adjustment of the object as by recording on photographic emulsions. Electro-optical crystals such as $\mathrm{Bi}_{12} \mathrm{SiO}_{20}$ (BSO) are appropriate for real time recording. They were already used for real-time holography [5-8] and speckle applications [9]. For the application to be described, the BSO crystal was biased with a transverse electric field $E_{0}$ in the 110 crystallographic direction. Illuminating the crystal with structured information in the T10 direction as indicated in fig. 1, a space-charge field is built-up, leading to a refractive index variation in the crystal via linear electro-optic effect. A phase vol-

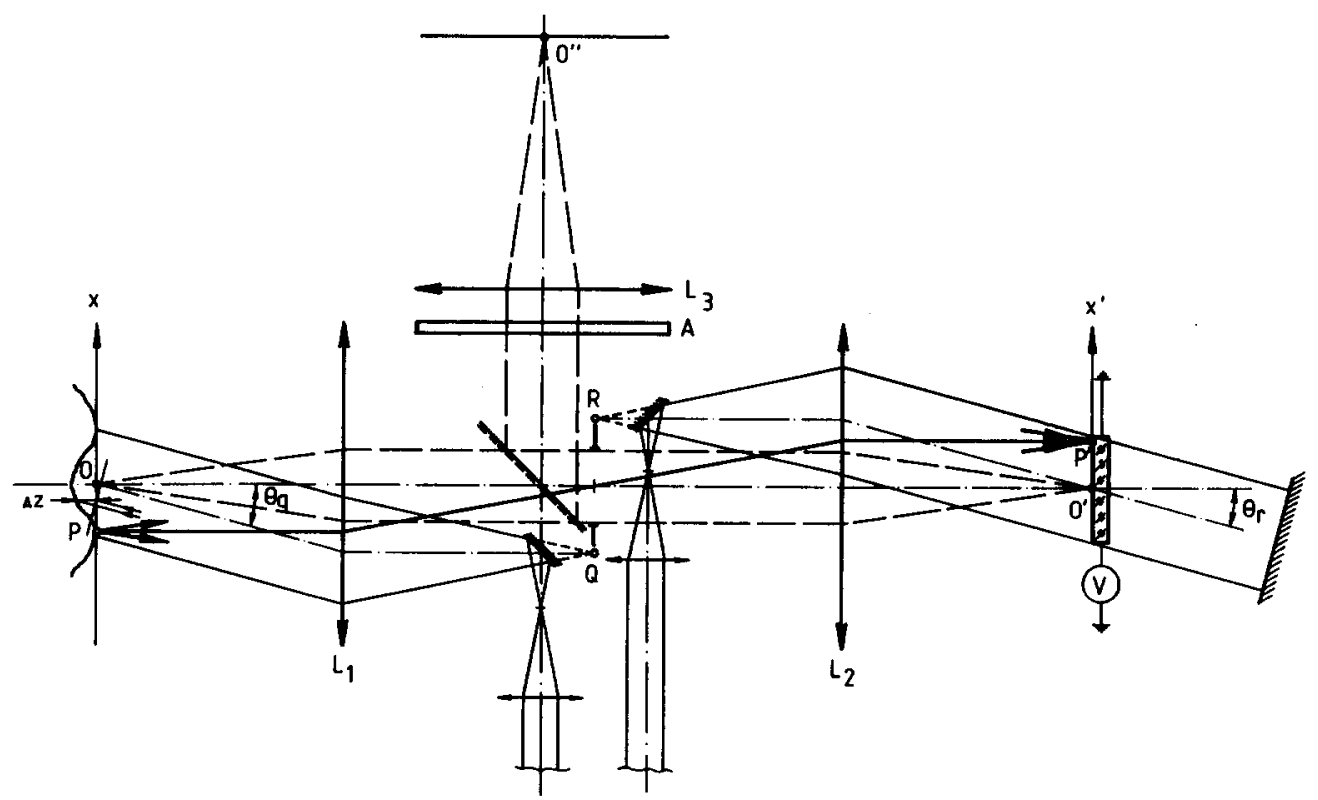

Fig. 1. Arrangement for real-time contouring. $L_{1}, L_{2}, L_{3}$ lenses. $Q, R$ illumination- and reference point sources. A polarization sheet. $\theta_{\mathrm{q}}, \theta_{\mathrm{r}}$ offset angles of illumination and reference plane waves.

0 030-4018/81/0000-0000/\$02.50 @ North-Holland Publishing Company 
ume hologram is created. Floading with uniform illumination leads to the erasure of the stored information by space-charge relaxation. Consequently, reading out with a recording wavelength is destructive.

For an optimised diffraction efficiency a BSO crystal of thickness $2.3 \mathrm{~mm}$ was chosen. For contour holography in rea.1 :ime a krypton laser was used lasing in neighbour wavelengths pairs appropriate for the experiments to be described.

\section{Principle of the contour line holography}

A brief analysis of the method adopted will be given. The experimental arrangement is shown schematically in fig. 1. A telecentric configuration is chosen to form the image of the object illuminated with a plane wave at an incident angle of $\theta_{\mathrm{q}}$. The image of the object is superimposed by the reference plane wave incident at an angle $\theta_{\mathrm{r}}$ in the image plane where the electro-optical crystal stores the information.

The complex amplitude immediately in front of a reference plane of the object can be written, using one dimensional notation only, as

$$
\begin{gathered}
A(x)=A_{0}(x) \exp \left(-\mathrm{i} \frac{2 \pi}{\lambda} x \sin \theta_{\mathrm{q}}\right) \\
\quad \mathrm{X} \exp \left[\mathrm{i} \frac{2 \pi}{\lambda}\left(1+\cos \theta_{\mathrm{q}}\right) \Delta z\right] .
\end{gathered}
$$

$A_{0}(x)$ describes the object as well as the optically rough surface. Furthermore, a perfect optical system is considered, hence $A(x) \rightarrow A^{\prime}\left(x^{\prime}\right)$. The interference of the complex amplitude of the image $A^{\prime}\left(x^{\prime}\right)$ of the object and the reference plane wave $A_{\mathrm{r}}^{\prime}\left(x^{\prime}\right)$ incident at an angle $\theta_{\mathrm{r}}$, as shown in fig. 1 , leads to an interference pattern in the crystal. The intensity of the fringe pattern in the crystal is proportional to $I_{1}^{\prime}\left(x^{\prime}, \lambda_{1}\right)$ for the wavelength $\lambda_{1}$ and $I_{2}^{\prime}\left(x^{\prime}, \lambda_{2}\right)$ for $\lambda_{2}$

$$
\begin{aligned}
& I_{1}^{\prime}\left(x^{\prime}, \lambda_{1}\right)=C_{1}\left\{\left|A_{\mathrm{r}}^{\prime}\left(x^{\prime}\right)\right|\left|A^{\prime}\left(x^{\prime}\right)\right|\right. \\
& \quad \times\left[\exp \left(\mathrm{i} \frac{2 \pi}{\lambda_{1}} L\right)+\exp \left(-\mathrm{i} \frac{2 \pi}{\lambda_{1}} L\right)\right] \\
& \left.\quad+\left|A_{\mathrm{r}}^{\prime}\left(x^{\prime}\right)\right|^{2}+\left|A^{\prime}\left(x^{\prime}\right)\right|^{2}\right\}
\end{aligned}
$$

and

$$
\begin{aligned}
& I_{2}^{\prime}\left(x^{\prime}, \lambda_{2}\right)=C_{2}\left\{\left|A_{\mathrm{I}}^{\prime}\left(x^{\prime}\right)\right|\left|A^{\prime}\left(x^{\prime}\right)\right|\right. \\
& \quad \times\left[\exp \left(\mathrm{i} \frac{2 \pi}{\lambda_{2}} L\right)+\exp \left(-\mathrm{i} \frac{2 \pi}{\lambda_{2}} L\right)\right] \\
& \left.\quad+\left|A_{\mathrm{r}}^{\prime}\left(x^{\prime}\right)\right|^{2}+\left|A^{\prime}\left(x^{\prime}\right)\right|^{2}\right\},
\end{aligned}
$$

$L=-\left(f_{1}^{\prime} / f_{2}^{\prime}\right) x^{\prime} \sin \theta_{\mathrm{q}}+x^{\prime} \sin \theta_{\mathrm{r}}-\left(1+\cos \theta_{\mathrm{q}}\right) \Delta z$,

$C_{1}$ and $C_{2}$ are constants.

The holograms for $\lambda_{1}$ and $\lambda_{2}$ are recorded simultaneously by using a laser emitting the two wavelengths.

The field distribution leads to a photoinduced change of the refractive index in the BSO crystal proportional to $I^{\prime}\left(x^{\prime}, \lambda_{1}, \lambda_{2}\right) \sim I_{1}^{\prime}\left(x^{\prime}, \lambda_{1}\right)+I_{2}^{\prime}\left(x^{\prime}, \lambda_{2}\right)$.

The complex transmission

$$
t^{\prime}\left(x^{\prime}, \lambda_{1}, \lambda_{2}\right)=\exp \left[i \beta I^{\prime}\left(x^{\prime}, \lambda_{1}, \lambda_{2}\right)\right]
$$

can be written as

$$
t^{\prime}\left(x^{\prime}, \lambda_{1}, \lambda_{2}\right)=1+\mathrm{i} \beta I^{\prime}\left(x^{\prime}, \lambda_{1}, \lambda_{2}\right),
$$

because

$$
\beta I^{\prime}\left(x^{\prime}, \lambda_{1}, \lambda_{2}\right) \ll \pi / 2 .
$$

The reconstructing wave can be either the conjugate reference wavefront of $\lambda_{1}$ or $\lambda_{2}$. The constructed image-wavefronts travel backwards through the telescope and are coupled out with a beamsplitter as shown in fig. 1. The reconstruction with the conjugate reference wave with wavelength $\lambda_{2}$ leads to an intensity pattern proportional to

$I=I_{0}+\widetilde{I} \cos \left(2 \pi / \lambda_{\text {res }}\right) L$

Constructive interference is obtained for $\left(2 \pi / \lambda_{\text {res }}\right) L=2 \pi m, \quad m=0, \pm 1, \pm 2 \ldots$

where $\lambda_{\text {res }}=\lambda_{1} \lambda_{2} /\left(\lambda_{1}-\lambda_{2}\right)$ can be considered as an equivalent wavelength.

For $m=1$

$\lambda_{\text {res }}=-\left(f_{1}^{\prime} / f_{2}^{\prime}\right) x^{\prime} \sin \theta_{\mathrm{q}}+x^{\prime} \sin \theta_{\mathrm{r}}-\left(1+\cos \theta_{\mathrm{q}}\right) \Delta z$. For $\left(f_{1}^{\prime} / f_{2}^{\prime}\right) \sin \theta_{\mathrm{q}}=\sin \theta_{\mathrm{r}}$, the separation of the contour lines is

$\Delta z=\lambda_{1} \lambda_{2} /\left(1+\cos \theta_{\mathrm{q}}\right)\left(\lambda_{1}-\lambda_{2}\right)$.

With the configuration chosen, the following practical advantages are achieved:

- the use of a grating for compensating the angles 

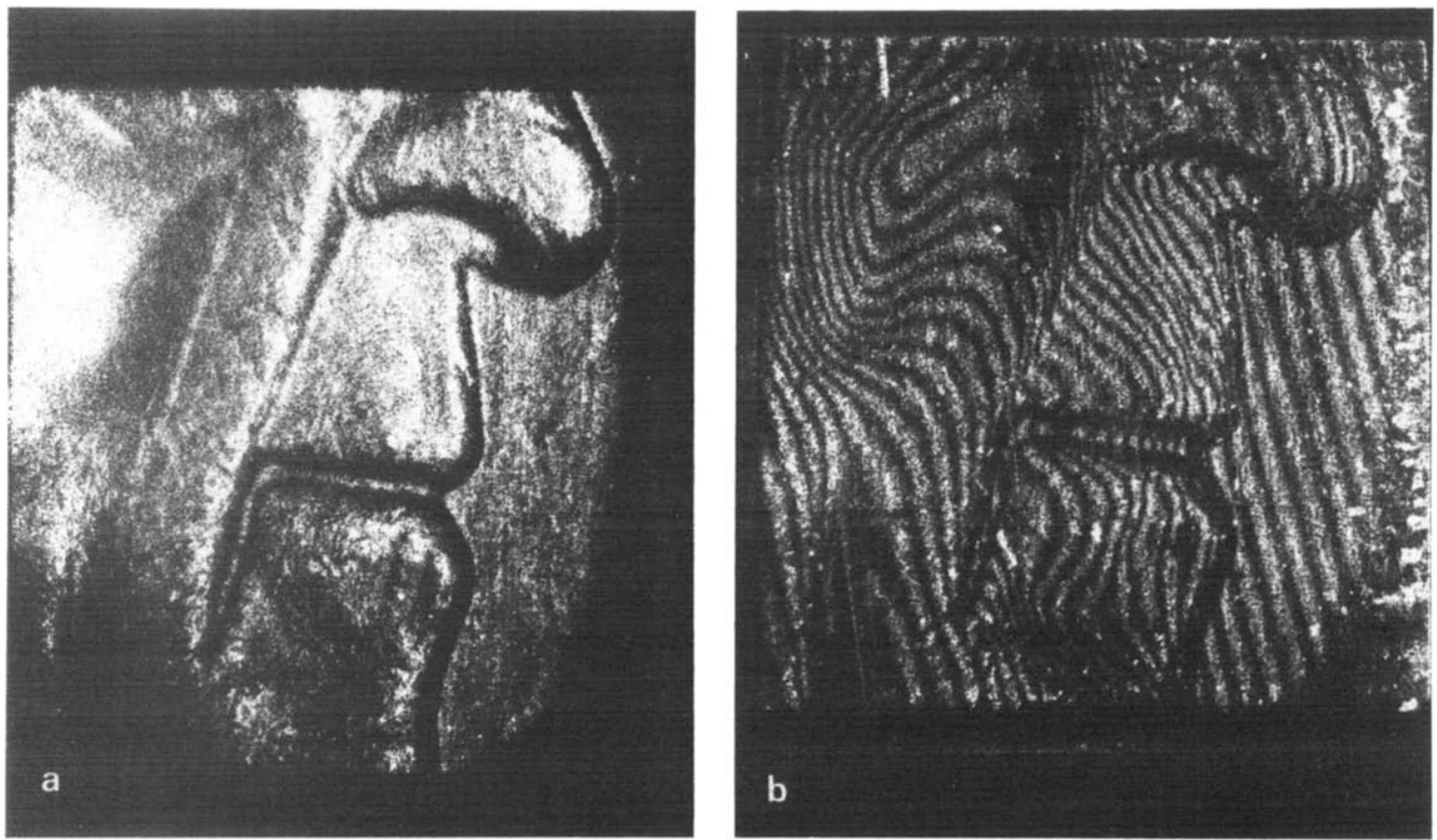

Fig. 2. (a) Image of part of a coin. (b) Real time contour lines of (a). $\lambda_{1}=520.83 \mathrm{~nm}, \lambda_{2}=530.87 \mathrm{~nm}$, contour line spacing is $13.88 \mu \mathrm{m}$.
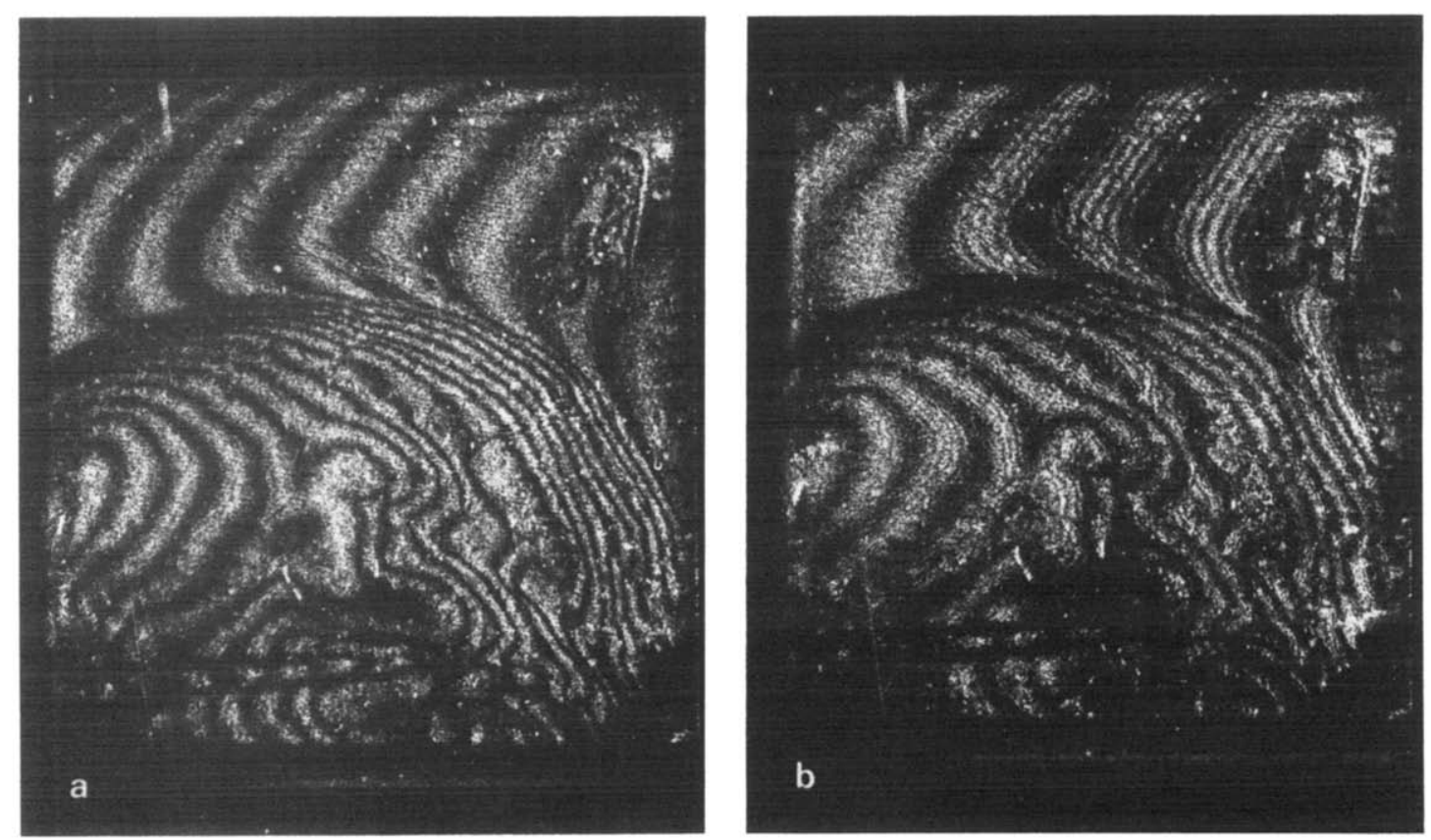

Fig. 3. (a) Contour lines on a metallic surface; contour line spacing $=13.88 \mu \mathrm{m}$. (b) Two sets of superimposed contour lines. Spacings are $18.45 \mu \mathrm{m}$ and $2.67 \mu \mathrm{m}$ with two pairs of laser lines $\left(\lambda_{1}=476.24 \mathrm{~nm}, \lambda_{2}=482.52 \mathrm{~nm}\right.$ and $\left.\lambda_{2}=482.52 \mathrm{~nm}, \lambda_{3}=530.87 \mathrm{~nm}\right)$. 
of the reference wave for the two wavelengths is avoided;

- the angles $\theta_{\mathrm{q}}$ and $\theta_{\mathrm{r}}$ can be small, for the telescope lenses to be used as collimators.

\section{Experimental results}

The experimental arrangement is shown in fig. 1 . As light source a krypton-ion laser was used. Pairs out of five laser lines were chosen. For a good diffraction efficiency of the BSO crystal a grating constant of 5 $\mu \mathrm{m}$ was envisaged, leading to $\theta_{\mathrm{q}}$ of 10 degrees, $f_{1}^{\prime} / f_{2}^{\prime}$ $=0.56 \ldots$ Furthermore, 6000 Volts were applied to the BSO crystal.

For a spacing of the contour lines of $13.88 \mu \mathrm{m}$, the two laser lines $\lambda_{1}=520.83 \mathrm{~nm}, \lambda_{2}=530.87 \mathrm{~nm}$ were selected. Fig. 2a shows an image of a coin, fig. $2 b$ the real-time contour lines. Fig. 3a shows another metallic object contoured with the same pair of wavelengths as in fig. 2b. For fig. $3 b$ three laser lines were used together, leading to two superimposed sets of contour lines. The wavelengths $\lambda_{1}=476.24 \mathrm{~nm}$ and $\lambda_{2}=$ $482.52 \mathrm{~nm}$ form contour lines with $18.45 \mu \mathrm{m}$ spacing, the wavelengths $\lambda_{2}=482.52 \mathrm{~nm}$ and $\lambda_{3}=530.87 \mathrm{~nm}$ the finer lines with a spacing of $2.67 \mu \mathrm{m}$. The writing power density on the crystal was roughly $200 \mu \mathrm{W}$ $\mathrm{cm}^{-2}$, the time constant for writing the hologram about $0.2 \mathrm{~s}$. Furthermore it should be mentioned, that for good image and fringe quality the appropriate orientation of a polarizer was found to be important.

\section{Conclusion}

Real time contouring with BSO crystals is found to be a very powerful tool for different applications for diffusing or transparent objects. The separation of the contour lines can be selected by choosing the appropriate wavelength pair with a properly chosen colour or interference filter. The adjustement of the object is carried out in real time.

We would like to thank the DFG for the financial support and B. Burger for carrying out some of the experimental work.

\section{References}

[1] K.A. Haines and B.P. Hildebrand, J. Opt. Soc. Amer. 57 (1967) 155.

[2] S. Zelenka and J.R. Varner, Appl. Optics 7 (1968) 2107.

[3] J.R. Varner, Appl. Optics 10 (1971) 212.

[4] W. Schmidt, A. Vogel and D. Preussler, Appl. Physics 1 (1973) 103.

[5] J.P. Herriau, J.P. Huignard and P. Aubourg, Appl. Optics 17 (1978) 1851.

[6] J.P. Huignard, J.P. Herriau, P. Aubourg and E. Spitz, Optics Lett. 4 (1979) 21.

[7] J.P. Huignard, J.P. Herriau, G. Rivet and P. Günter, Optics Lett. 5 (1980) 102.

[8] A. Marrakchi, J.P. Huignard and J.P. Herriau, Optics Comm. 34 (1980) 15.

[9] H.J. Tiziani, K. Leonhardt and J. Klenk, Optics Comm. 34 (1980) 327. 